\title{
Licht-Kunst mit therapeutischen Effekten
}

Mit seiner Lichtinstallation „Memory Code" im 2012 neu errichteten Pflegewohnhaus Simmering hat der Künstler Friedrich Biedermann nicht nur ein bemerkenswertes Kunstobjekt in einer geriatrischen Einrichtung geschaffen - die Lichteffekte wirken zudem auch positiv auf das Wohlbefinden der Bewohner.

Für Mediziner ist das plausibel, schließlich gibt es zahlreiche Belege für den gezielten therapeutischen Einsatz von Licht in unterschiedlichen Formen. "Licht hat einen stimulierenden Einfluss auf Menschen", erklärt OA Dr. Norbert Embacher, Facharzt für Neurologie am LKH St. Pölten. „Regelmäßig und in der richtigen Stärke angewendet, kann sich Licht positiv auf Stimmung und Wohlbefinden auswirken. Davon können nicht nur die Patienten oder Bewohner einer medizinischen oder pflegerischen Einrichtung profitieren, sondern auch die Mitarbeiter."

Die Lichtinstallation "Memory Code“ bildet das Herzstück eines in dieser Form in den Wiener Geriatriezentren bisher einzigartigen multikonfessionel-

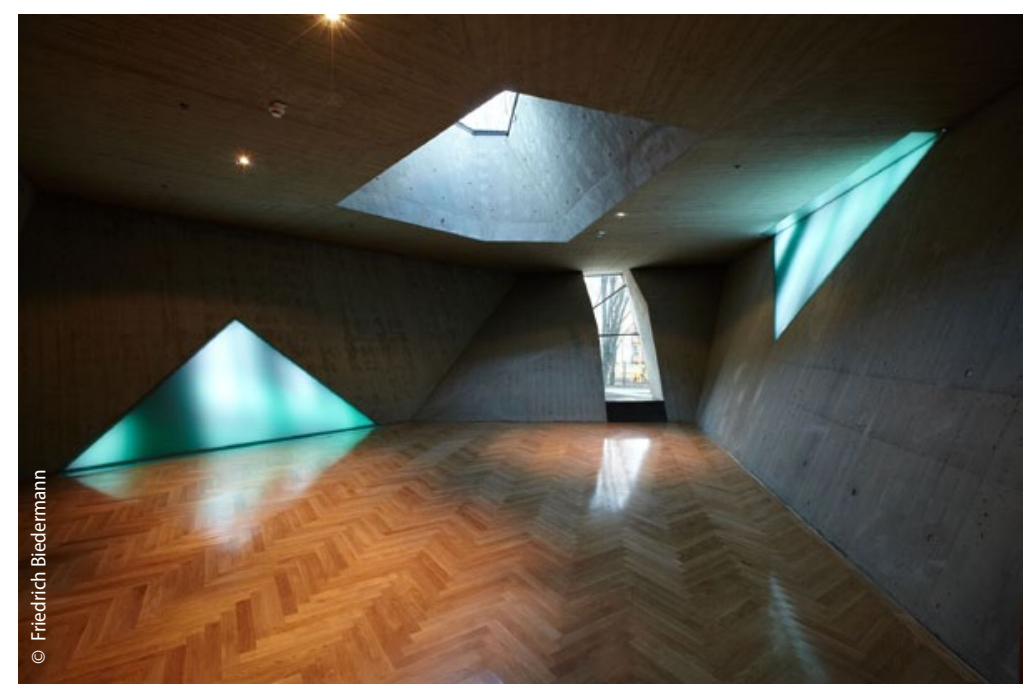

Abb. $1 \Delta$ Lichteffekte können in besonderem Maße auch Menschen mit dementiellen Erkrankungen positiv beeinflussen. len Meditationsraums, den Biedermann in Zusammenarbeit mit Josef Weichenberger Architects \& Partner kreiert hat und der für Veranstaltungen, Regeneration und Therapie genutzt wird. "Mein Ziel war es, einen Ort zu schaffen, der positiv auf die Betrachter wirkt", sagt Friedrich Biedermann. Die Lichtinstallation entsteht durch das Zusammenspiel zweier Lichtdreiecke, auf denen Stimmungsfilme abgespielt werden, es entsteht eine Beziehung zu den Betrachtern und ihrer Erinnerung. „Dieser Raum bietet die Möglichkeit, Ruhe zu finden und zu verweilen. Gleichzeitig macht die Installation neugierig, man will wissen, was als Nächstes im Lichtspiel kommt." Der beruhigende Wechsel von Licht und Farbe hat darüber hinaus einen therapeutischen Effekt.

Von besonderer Bedeutung, gerade in einem geriatrischen Zentrum: Neben dem nachgewiesenen Nutzen des therapeutischen Einsatzes von Licht bei Depressionen können Lichteffekte in besonderem Maß auch Menschen mit dementiellen Erkrankungen positiv be-

einflussen, die häufig gestörte circadiane Rhythmen aufweisen und etwa zur Zeit des Sonnenuntergangs besonders wach, aber auch unruhig werden. „Hier kann das richtige Licht einen ausgleichenden und beruhigenden Einfluss haben", so der Neurologe.

Wesentlich ist auch die Art des verwendeten Lichts, erklärt Embacher. So zeigte sich bei Menschen mit Depressionen, dass mit weißem Licht mit einer höheren Lichtstärke ein besserer Effekt zu erzielen ist als mit gedämpftem Licht. „Bei Demenzpatienten wiederum wirkt blaues Licht positiver als rotes", so der Experte.

Wünschenswert wäre, so Friedrich Biedermann, die begleitenden medizinischen Effekte seiner Lichtkunst systematisch zu evaluieren. „Dann könnten solche Installationen noch gezielter in geriatrischen Settings zum Nutzen der Bewohner eingesetzt werden." Eine Einschätzung, die auch in der internationalen Debatte zum Thema geteilt wird. So sieht etwa eine Untersuchung der britischen Loughborough University wichtige Perspektiven für eine Zusammenarbeit zwischen Medizin und Gestaltern, um maßgeschneiderte Lichtlösungen für spezielle Indikationen in der Altenbetreuung zu entwickeln. Es sei weitere Forschung notwendig, um therapeutische Effekte von Licht gezielt integrieren zu können, so das britische Forscherteam.

Informationen: E-Mail: office@friedrichbiedermann.com, Internet: http://friedrichbiedermann.xarch.at/wordpress/ ?page_id=568

\section{Referenz:}

1. Shikder et al., Therapeutic lighting design for the eldery: A review, Perspectives in Public Health 132.6 (2012): 282-291 